\title{
In the World of Bladder Tumors: Size Does Matter
}

\author{
Justin Loloi ${ }^{\mathrm{a}}$, Jordan L. Allen ${ }^{\mathrm{a}}$, Amber Schilling ${ }^{\mathrm{b}}$, Christopher Hollenbeak ${ }^{\mathrm{c}}$, Suzanne B. Merrill ${ }^{\mathrm{a}}$, \\ Matthew G. Kaag a and Jay D. Raman ${ }^{\mathrm{a}, *}$ \\ ${ }^{a}$ Division of Urology, Department of Surgery, Penn State Milton S. Hershey Medical Center, \\ The Pennsylvania State University College of Medicine, Hershey, PA, USA \\ ${ }^{\mathrm{b}}$ Division of Outcomes Research and Quality, Department of Surgery, The Pennsylvania State University \\ College of Medicine, Hershey, PA, USA \\ ${ }^{\mathrm{c}}$ Department of Health Policy and Administration, The Pennsylvania State University, State College, PA, USA
}

Received 13 January 2020

Accepted 15 April 2020

Pre-press 7 May 2020

Published 11 June 2020

\begin{abstract}
.
BACKGROUND: Transurethral resection of bladder tumor (TURBT) is fundamental to the diagnosis and management of bladder cancer. The impact of tumor size on perioperative outcomes is seemingly intuitive albeit incompletely defined.

OBJECTIVE: To compare outcomes following TURBT of small, medium, and large tumors to determine if larger tumors truly resulted in a greater degree of complications.

METHODS: The National Surgical Quality Improvement Project (NSQIP) Participant Use File (PUF) was queried to extract all TURBT cases performed from 2011-2015. CPT codes 52234 (small), 52235 (medium), and 52240 (large) were used to stratify the data into three cohorts. Outcomes of interest included any complications, hospital length of stay (LOS), reoperation within 30-days, 30-day readmission, and mortality.

RESULTS: 17,839 patients who underwent TURBT were included. $44 \%$ had small $(n=7,805), 35 \%$ had medium $(n=6,240)$, and $21 \%$ had large tumors $(n=3,794)$. Univariate analysis revealed significant differences in complications, length of stay, reoperation rate, readmission at 30-days, and mortality when stratifying TURBT by tumor size $(p<0.0001)$. In the multivariable regression model, medium and large tumors were associated with significantly greater odds of a postoperative complication ( $\mathrm{OR}=1.37$ and $1.64 ; p<0.0001)$, reoperation ( $\mathrm{OR}=1.33$ and $1.52 ; p=0.019$ and $p=0.002)$, readmission at 30-days ( $\mathrm{OR}=1.27$ and $1.56 ; p=0.001$ and $p<0.0001)$, and death $(\mathrm{OR}=1.65$ and $2.59 ; p=0.015$ and $p<0.0001)$ compared to smaller tumors.

CONCLUSIONS: Larger tumor size $(>5 \mathrm{~cm})$ is associated with greater length of stay, reoperation, readmission, and death following TURBT. Patients should be counseled appropriately and likely warrant vigilant observation prior to and following hospital discharge.
\end{abstract}

Keywords: Bladder, TURBT, cancer, tumor, size, resection, complication, postoperative

\section{INTRODUCTION}

*Correspondence to: Jay D. Raman, M.D., F.A.C.S., Penn State Health Milton S. Hershey Medical Center, Division of Urology, 500 University Drive, BMR Building c4830B, Hershey, PA 170330850, USA. Tel.: +1 717531 6979; Fax: +1 717531 4475; E-mail: jraman@pennstatehealth.psu.edu.
Bladder cancer (BC) is a commonly occurring genitourinary cancer with significant morbidity and mortality in both men and women [1]. Smoking and exposure to environmental agents are known risk factors for the development of this disease [2]. 
$\mathrm{BC}$ is a heterogeneous entity with a spectrum of histopathology which dictates its natural history and biologic aggressiveness [3, 4]. Accurate assessment of disease characteristics is therefore paramount in management.

Cystoscopy with transurethral resection (TUR) is a fundamental procedure essential not only for diagnosis and staging but also in the management of suspected bladder tumors. For non-muscle invasive cancers, TUR may be curative although adjuvant intravesical therapies often are necessary. Radical cystectomy remains the "gold-standard" for treatment of muscle invasive bladder cancer. Trimodality therapy, which includes transurethral resection (TUR), radiation, and systemic chemotherapy, represents a well-established alternative to radical cystectomy and has been shown to have comparable long-term outcomes in appropriately selected patients $[2,5]$.

Given the importance of transurethral resection of bladder tumors (TURBT), it is essential to recognize some of the adverse sequelae of the procedure which may impact convalescence and recovery. In this regard, the National Surgical Quality Improvement Program (NSQIP) provides useful data pertaining to index procedures and associated perioperative morbidity. Here, we evaluate peri-operative outcomes of TURBT focusing on the impact of size of resection (as determined by CPT codes).

\section{MATERIALS AND METHODS}

The National Surgical Quality Improvement Project (NSQIP) Participant Use File (PUF) was queried to extract all TURBT cases performed from 2011-2015. Given the nature of this article and the use of the NSQIP, IRB approval was deemed exempt. CPT codes 52234 (small, defined as $<2 \mathrm{~cm}$ resection), 52235 (medium, defined as $2-5 \mathrm{~cm}$ ), and 52240 (large, defined as $>5 \mathrm{~cm}$ ) were queried to stratify the data into three cohorts. Outcomes of interest included the presence of any post-surgical complications, hospital length of stay (LOS), reoperation within 30-days, 30-day readmission, and mortality.

Inclusion and exclusion criteria were limited. All patients undergoing TURBT from 2011-2015 with use of CPT codes 52234 (TURBT $<2 \mathrm{~cm}$ ), 52235 (TURBT $2-5 \mathrm{~cm}$ ), and 52240 (TURBT $>5 \mathrm{~cm}$ ) were included in the study. The NSQIP dataset permitted evaluating each procedure as a unique event, and therefore a prior history of TUR or restaging TUR procedures could not be identified or segregated for analysis. Patients undergoing bladder cancer procedures with CPT codes of 52224 (TURBT $<0.5 \mathrm{~cm}$ ) and 52214 (cystoscopy with fulguration) were excluded from the study.

The NSQIP includes information on medical comorbidities, including any history of diabetes, hypertension, pulmonary comorbidities, cardiac comorbidities, and American Society of Anesthesiologists (ASA) class. A pulmonary comorbidity was defined as a history of severe chronic obstructive pulmonary disease (COPD), requiring ventilatorassisted respiration within 48 hours before surgery, or current pneumonia. Cardiac comorbidity was defined as a history of congestive heart failure (CHF, within 30 days before admission), myocardial infarction (within six months before admission), cardiac surgery, percutaneous coronary intervention, or angina (within one month before admission). ASA class $\geq 3$ corresponds to severe systemic disease. The comorbidity number represents the sum of these comorbidities within the American College of Surgeons NSQIP surgical risk calculator and is distinct from other published measures of comorbidities such as the Charlson and Elixhauser indices.

ANOVA was used to detect statistical significance between continuous variables across the three cohorts and chi-square tests were used for binary variables. Linear and logistic regressions were utilized to control for potential confounders. Significance was set at a $p$ value of $<0.05$.

\section{RESULTS}

From 2011-2015, 17,839 patients who underwent TURBT were included with the distribution of tumor sizes summarized in Fig. 1. Table 1 displays the demographics data for this cohort. In aggregate, the mean age of the study population was 71 years with $75 \%$ being male gender and $71 \%$ of Caucasian race. A significant proportion of patients had baseline comorbid conditions most notably hypertension

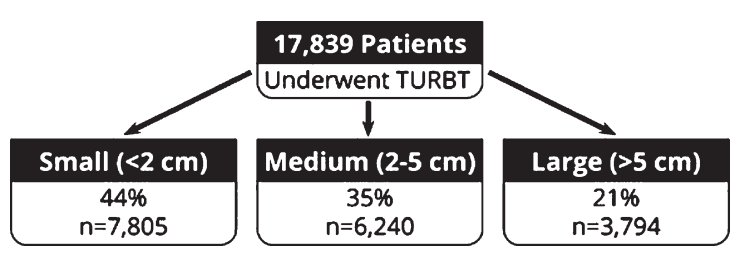

Fig. 1. Cohort stratification by tumor resection size. 
Table 1

Cohort Demographics

\begin{tabular}{|c|c|c|c|c|c|}
\hline \multirow[t]{2}{*}{ Variable } & \multicolumn{4}{|c|}{ Tumor } & \multirow[t]{2}{*}{$p$-value } \\
\hline & $\begin{array}{c}\text { Overall } \\
(n=17839)\end{array}$ & $\begin{array}{c}\text { Small } \\
(n=7805)\end{array}$ & $\begin{array}{c}\text { Medium } \\
(n=6240)\end{array}$ & $\begin{array}{c}\text { Large } \\
(n=3794)\end{array}$ & \\
\hline Age (mean, years) & 71 & 71 & 70.9 & 71.3 & 0.2305 \\
\hline $18-64$ & $26.20 \%$ & $25.90 \%$ & $26.80 \%$ & $25.90 \%$ & \\
\hline $65-74$ & $31.50 \%$ & $31.50 \%$ & $31.90 \%$ & $30.90 \%$ & \\
\hline $75-79$ & $16.40 \%$ & $16.90 \%$ & $16.00 \%$ & $16.00 \%$ & \\
\hline$\geq 80$ & $25.90 \%$ & $25.70 \%$ & $25.40 \%$ & $27.20 \%$ & \\
\hline Race/Ethnicity & & & & & $<0.0001$ \\
\hline White & $71.20 \%$ & $61.80 \%$ & $78.00 \%$ & $79.50 \%$ & \\
\hline Black & $4.40 \%$ & $3.90 \%$ & $4.20 \%$ & $5.60 \%$ & \\
\hline Hispanic & $4.20 \%$ & $3.80 \%$ & $4.20 \%$ & $4.80 \%$ & \\
\hline Other & $3.00 \%$ & $3.10 \%$ & $3.00 \%$ & $3.00 \%$ & \\
\hline Unknown & $17.20 \%$ & $27.40 \%$ & $10.50 \%$ & $7.10 \%$ & \\
\hline Sex & & & & & $<0.0001$ \\
\hline Male & $75.40 \%$ & $74.00 \%$ & $76.50 \%$ & $76.70 \%$ & \\
\hline Female & $24.60 \%$ & $26.00 \%$ & $23.50 \%$ & $23.50 \%$ & \\
\hline BMI (mean, $\mathrm{kg} / \mathrm{m}^{2}$ ) & 27.77 & 27.83 & 27.83 & 27.56 & 0.1142 \\
\hline Underweight $(\mathrm{BMI}<18.5)$ & $7.00 \%$ & $6.40 \%$ & $7.20 \%$ & $7.70 \%$ & \\
\hline Normal $(18.5 \leq \mathrm{BMI}<25)$ & $24.70 \%$ & $24.60 \%$ & $23.90 \%$ & $26.40 \%$ & \\
\hline Overweight $(2 \overline{5} \leq \mathrm{BMI}<30)$ & $36.10 \%$ & $37.10 \%$ & $36.10 \%$ & $34.10 \%$ & \\
\hline Obese $(\mathrm{BMI} \geq 3 \overline{0})$ & $32.20 \%$ & $31.90 \%$ & $32.80 \%$ & $31.70 \%$ & \\
\hline Class I & $20.00 \%$ & $20.00 \%$ & $20.50 \%$ & $19.00 \%$ & \\
\hline Class II & $7.90 \%$ & $7.60 \%$ & $8.20 \%$ & $7.90 \%$ & \\
\hline Class III & $4.30 \%$ & $4.20 \%$ & $4.10 \%$ & $4.90 \%$ & \\
\hline \multicolumn{6}{|c|}{ Comorbidities/Pre-Surgical Risk Factors } \\
\hline Diabetes & $22.40 \%$ & $21.80 \%$ & $23.10 \%$ & $22.50 \%$ & 0.182 \\
\hline Non-Insulin Dependent & $15.10 \%$ & $14.90 \%$ & $15.70 \%$ & $14.50 \%$ & \\
\hline Insulin-Dependent & $7.30 \%$ & $6.90 \%$ & $7.40 \%$ & $8.00 \%$ & \\
\hline Smoking & $19.80 \%$ & $17.60 \%$ & $20.00 \%$ & $24.00 \%$ & $<0.0001$ \\
\hline COPD & $10.20 \%$ & $9.60 \%$ & $10.10 \%$ & $11.60 \%$ & 0.004 \\
\hline $\mathrm{CHF}$ & $1.10 \%$ & $0.90 \%$ & $1.00 \%$ & $1.40 \%$ & 0.09 \\
\hline HTN & $63.00 \%$ & $62.50 \%$ & $63.50 \%$ & $63.40 \%$ & 0.43 \\
\hline Acute Renal Failure or Dialysis & $1.20 \%$ & $1.00 \%$ & $1.10 \%$ & $1.70 \%$ & 0.002 \\
\hline ARF & $0.50 \%$ & $0.30 \%$ & $0.40 \%$ & $1.00 \%$ & \\
\hline Dialysis & $0.80 \%$ & $0.70 \%$ & $0.80 \%$ & $0.80 \%$ & \\
\hline Bleeding Disorder & $4.40 \%$ & $4.40 \%$ & $4.60 \%$ & $4.10 \%$ & 0.413 \\
\hline Transfusion Pre-Op & $1.40 \%$ & $0.60 \%$ & $1.30 \%$ & $3.30 \%$ & $<0.0001$ \\
\hline Comorbidity Number & 1.56 & 1.5 & 1.58 & 1.64 & $<0.0001$ \\
\hline 0 & 17.50 & $18.80 \%$ & $16.90 \%$ & $15.70 \%$ & \\
\hline 1 & $34.50 \%$ & $35.50 \%$ & $34.20 \%$ & $32.90 \%$ & \\
\hline 2 & $28.4 \%$ & $27.00 \%$ & $29.00 \%$ & $30.30 \%$ & \\
\hline 3 & $19.60 \%$ & $18.70 \%$ & $19.90 \%$ & $21.10 \%$ & \\
\hline Any & $82.50 \%$ & $81.20 \%$ & $83.10 \%$ & $84.30 \%$ & \\
\hline ASA Class & 2.64 & 2.6 & 2.66 & 2.72 & $<0.0001$ \\
\hline 1. No Disturbance & $2.30 \%$ & $3.00 \%$ & $1.90 \%$ & $1.70 \%$ & \\
\hline 2. Mild Disturbance & $37.10 \%$ & $39.50 \%$ & $36.50 \%$ & $33.30 \%$ & \\
\hline 3. Severe Disturbance & $54.40 \%$ & $52.40 \%$ & $\% 55.60$ & $56.60 \%$ & \\
\hline 4. Life Threatening & $6.10 \%$ & $5.20 \%$ & $\% 6.00$ & $8.40 \%$ & \\
\hline 5. Morilbund & $0.00 \%$ & $0.00 \%$ & $0.00 \%$ & $0.10 \%$ & \\
\hline
\end{tabular}

(63\%), diabetes mellitus (22\%), and COPD (10\%). Patients with larger tumors were more likely to have a greater number of comorbidities, higher baseline ASA score, as well as higher rates of acute renal insufficiency, COPD, and preoperative transfusion requirement (Table 1$)$.

Table 2 summarizes the univariate analysis between the three cohorts with respect to post- operative events. Approximately $6 \%$ of patients experienced a complication following TURBT. Complications included pneumonia, UTI, thromboembolic events, sepsis, transfusion, acute renal insufficiency, and death. Analysis revealed a significant difference in number of complications, length of stay, reoperation rate, re-admission at 30 days, and mortality between the three cohorts ( $p$ for all 
Table 2

Univariate analysis of postoperative events based on tumor size

\begin{tabular}{lccccc}
\hline Variable & \multicolumn{4}{c}{ Tumor } & $p$-value \\
\cline { 2 - 5 } & $\begin{array}{c}\text { Overall } \\
(n=17839)\end{array}$ & $\begin{array}{c}\text { Small } \\
(n=7805)\end{array}$ & $\begin{array}{c}\text { Medium } \\
(n=6240)\end{array}$ & $\begin{array}{c}\text { Large } \\
(n=3794)\end{array}$ & \\
\hline Complication Number & 0.08 & 0.05 & 0.08 & 0.12 & $<0.0001$ \\
0 & $93.90 \%$ & $96.00 \%$ & $93.60 \%$ & $90.20 \%$ & \\
1 & $5.00 \%$ & $3.40 \%$ & $5.40 \%$ & $7.70 \%$ & \\
2 & $0.80 \%$ & $0.40 \%$ & $0.80 \%$ & $1.70 \%$ & \\
$\geq 3$ & $0.30 \%$ & $0.20 \%$ & $0.30 \%$ & $0.30 \%$ & \\
Any & $6.10 \%$ & $4.00 \%$ & $6.40 \%$ & $9.80 \%$ & $<0.0001$ \\
Operative Time (means, minutes) & 33.13 & 24.7 & 33.56 & 49.76 & $<0.0001$ \\
$\quad \leq 15$ & $24.60 \%$ & $37.90 \%$ & $18.30 \%$ & $7.50 \%$ & \\
$16-25$ & $25.80 \%$ & $29.40 \%$ & $28.00 \%$ & $14.80 \%$ & \\
$26-40$ & $23.80 \%$ & $19.60 \%$ & $27.60 \%$ & $26.20 \%$ & \\
$>40$ & $25.80 \%$ & $13.10 \%$ & $26.00 \%$ & $51.50 \%$ & \\
LOS (mean, days) & 0.85 & 0.54 & 0.84 & 1.51 & $<0.0001$ \\
Reoperation & $2.30 \%$ & $1.70 \%$ & $2.50 \%$ & $3.40 \%$ & $<0.0001$ \\
Readmitted & $7.30 \%$ & $5.40 \%$ & $7.60 \%$ & $10.70 \%$ & $<0.0001$ \\
Died & $1.10 \%$ & $0.50 \%$ & $1.10 \%$ & $2.20 \%$ & $<0.0001$ \\
\hline
\end{tabular}

$<0.0001$ ). Diabetes mellitus, smoking, CHF, and acute renal failure were associated with presence of a post-operative complication. CHF and acute renal failure were correlated with hospital LOS. Presence of a bleeding disorder was associated with reoperation following TURBT. Additionally, CHF along with acute renal failure were correlated with 30-day readmission and death.

A multivariate regression model was constructed focusing on the index variable of tumor size on key perioperative outcomes. In that regard, the abovementioned baseline comorbidity differences were controlled. Our analysis noted that tumors belonging to the medium or large cohort were associated with a significantly greater odds of a postoperative complication $(\mathrm{OR}=1.37$ and $1.64 ; p<0.0001)$, reoperation $(\mathrm{OR}=1.33$ and $1.52 ; p=0.019$ and $p=0.002)$, readmission at 30 days $(\mathrm{OR}=1.27$ and $1.56 ; p=0.001$ and $p<0.0001)$, and death $(\mathrm{OR}=1.65$ and $2.59 ; p=0.015$ and $p<0.0001)$ compared to smaller tumors (Table 3). In addition, we also found that large tumors were associated with a significantly longer LOS than were smaller tumors ( 0.40 days longer; $p<0.0001)$. Predictably, larger tumors were also associated with a longer operative time.

\section{DISCUSSION}

The aim of this study was to use a large database focusing on surgical complications to better determine the risks of TURBT specifically when stratified by size of resection. We found that larger tumors $(>5 \mathrm{~cm})$ are associated with a higher likelihood of developing postoperative complications (ex: UTI, transfusion, acute renal failure, blood clot, sepsis). Additionally, LOS, reoperation, readmission, and mortality following TURBT were also found to be significantly greater in the large tumor cohort. These results implicate that (not surprisingly) tumor size and resection volume impacts surgical recovery.

Table 3

Multivariable regression model of postoperative events between groups based on tumor size

\begin{tabular}{|c|c|c|c|c|c|}
\hline \multirow{3}{*}{ Post-op complication } & \multirow{2}{*}{$\frac{\text { Small }(<2 \mathrm{~cm})}{\text { Reference }}$} & \multicolumn{2}{|c|}{ Medium $(2-5 \mathrm{~cm})$} & \multicolumn{2}{|c|}{ Large $(>5 \mathrm{~cm})$} \\
\hline & & $\mathrm{OR}=1.37$ & $p<0.0001$ & $\mathrm{OR}=1.64$ & $p<0.0001$ \\
\hline & & $1.17-1.61$ & (95\% confidence) & $1.38-1.95$ & (95\% confidence) \\
\hline \multirow{2}{*}{ Reoperation } & Reference & $\mathrm{OR}=1.33$ & $p=0.019$ & $\mathrm{OR}=\mathbf{1 . 5 2}$ & $p=0.002$ \\
\hline & & $1.05-1.70$ & (95\% confidence) & $1.16-2.00$ & (95\% confidence) \\
\hline \multirow{2}{*}{ Readmission within 30 days } & Reference & $\mathrm{OR}=1.27$ & $p=0.001$ & $\mathrm{OR}=\mathbf{1 . 5 6}$ & $p<0.0001$ \\
\hline & & $1.10-1.46$ & (95\% confidence) & $1.34-1.82$ & (95\% confidence) \\
\hline \multirow{2}{*}{ Death within 30 days } & Reference & $\mathrm{OR}=1.65$ & $p=0.0001$ & $\mathrm{OR}=2.59$ & $p=0.0001$ \\
\hline & & $1.10-2.48$ & (95\% confidence) & $1.72-3.92$ & (95\% confidence) \\
\hline \multirow[t]{2}{*}{ Length of stay } & Reference & +0.14 days & $p=0.1391$ & +0.40 days & $p<0.0001$ \\
\hline & & $-0.04-0.32$ & (95\% confidence) & $0.17-0.62$ & ( $95 \%$ confidence) \\
\hline
\end{tabular}


Therefore, patients should be counseled appropriately prior to undergoing TURBT on the possibility of a longer period of observation prior to discharge as well as the potential for readmission depending on extent of TUR.

TURBT remains the first-line option in the diagnosis and treatment of bladder cancer as well as management of recurrences. Although TURBT is considered an endoscopic and often ambulatory procedure it is not without complications [6]. A large database analysis evaluating morbidity of urological procedures found a TURBT-related complication rate of $11 \%$ [7]. A prospective evaluation of all TURBTs performed during a one-year period at an academic center revealed a complication rate of 5.8\%. These complications included hematuria requiring transfusion and bladder perforation [8]. Our analysis focusing on over 15,000 TURBT procedures in NSQIP noted a similar complication rate of approximately $6 \%$.

Specifically, regarding immediate postoperative complications following TURBT, Gregg et al. found the overall complication rate to be $8.1 \%$, with the most common complications being pain/spasm (3\%), retention $(2.8 \%)$, and infection $(2.1 \%)$. These authors noted that a prior complication and single tumor, but no other patient or tumor-specific characteristic, to be associated with developing a postoperative complication [9]. Similarly, operative duration, has been shown to play an important prognostic role following TURBT. That is, increased operative time is associated with postoperative complication, even after controlling for patient demographic data and tumor characteristics [10].

There is relative paucity of data directly associating tumor size found on TURBT to the presence of postoperative complications. Ghali et al. conducted a retrospective review of TURBTs performed at a single-center in order to evaluate factors associated with unplanned hospital return [11]. They found an unplanned hospital return rate of $10.9 \%$, most commonly due to hematuria. Their analysis demonstrated that tumor size had no association to an unplanned hospital return following bladder tumor resection. Other authors, however, have observed that tumor size may be a potential risk factor for 30-day and 90-day mortality following TURBT. In their review of the NSQIP database, Hollenbeck et al. found that larger tumors seemed to be associated with a higher postoperative mortality [12]. Our study representing a more contemporary cohort builds on this earlier observation by noting that larger tumors, compared to their smaller counterparts, are associated with a greater risk of postsurgical complications, LOS, reoperation, readmission, and mortality following TURBT.

Although LOS was only extended approximately 0.40 days, these observations may have bearing in a healthcare environment where endoscopic procedures are increasingly performed in outpatient setting. Collectively, we believe these findings underscore the importance of using tumor size as a discussion point for proper informed consent conversation regarding expectations following surgery. This study is not without limitations. The actual sizes of the tumors were unknown due to the nature of categorizing by CPT codes. Therefore, the analysis is subject to variability with respect to how urologists coded specific procedures with potential subjectivity in assessment. As the main focus of this paper is the effect of tumor size on TURBT complications, we did not include the use of intravesical therapy although this may also contribute to complications in a small percentage of patients. Furthermore, reimbursement is stratified according to CPT code and so tumor size is fairly subjective and may be biased by this incentive-based model. Indeed, there is no pathologic information pertaining to the resection and these factors may impact the quality and thoroughness of resection. Furthermore, with regards to reoperation, there may be inadvertent capture of planned repeat TURBTs for further staging of the disease. This may be pertinent to patients with $\mathrm{T} 1$ disease or those with larger tumors who have anticipated staged resections. Logistic regression modeling allowed us to assess the impact of multiple variables in the same model. Logistic regression modeling assumes that the selected co-variates are independent of one another. Independent variables in Table 1 were run in logistic regression models against each of the dichotomous variables listed in Table 3. Potential confounders in our multivariate model are the relationship between operative time and tumor size and the association of diabetes with obesity. We acknowledge these as potential confounders but determined that these were not significant enough to preclude logistic regression modeling. Finally, these data do not permit understanding the granularity of cases with regards to therapeutic versus palliative surgery or potential emergent nature of resection. Nonetheless, despite these limitations, we feel the current study has identified and filled a gap in the literature through easily measured metrics, although further study and data is warranted. 


\section{CONCLUSION}

Postoperative care following bladder cancer TUR is heterogenous, particularly with respect to tumor size. Although postoperative protocols and clinical practice remain surgeon-dependent, the data presented suggests that Urologists should counsel patients and set clear expectations for the potential of a prolonged duration of hospital stay and occurrence of complication depending on tumor size found on resection. Setting clear expectations may have a positive effect on patient satisfaction. Future studies will focus on the financial significance of these findings and effects on patient-satisfaction following TURBT.

\section{ACKNOWLEDGMENTS}

The authors have no acknowledgments.

\section{FUNDING}

The Ken and Bonnie Shockey Fund for Urologic Oncology Research at Penn State Health.

\section{AUTHOR CONTRIBUTIONS}

JL: drafting of the manuscript, substantial contributions to the conception or design of the work or the acquisition, analysis, or interpretation of data for the work, drafting the work for important intellectual content; JLA: data collection and analysis and manuscript creation, substantial contributions to the conception or design of the work or the acquisition, analysis, or interpretation of data for the work; AS, $\mathrm{CH}$ : statistical analysis and manuscript creation, substantial contributions to the conception or design of the work or the acquisition, analysis, or interpretation of data for the work, drafting the work for important intellectual content; SM, MK: data collection and analysis and manuscript creation, substantial contributions to the conception or design of the work or the acquisition, analysis, or interpretation of data for the work; JDR: oversight of all aspects of project including construction of protocol, data collection and analysis and manuscript creation, substantial contributions to the conception or design of the work or the acquisition, analysis, or interpretation of data for the work, drafting the work and revising it critically for important intellectual content.

\section{CONFLICT OF INTEREST}

The authors declare no potential conflicts of interest with respect to research, authorship and/or publication of this article

\section{REFERENCES}

[1] Kirkali Z, Chan T, Manoharan M, Algaba F, Busch C, Cheng $\mathrm{L}$, et al. Bladder cancer: epidemiology, staging and grading, and diagnosis. Urology. 2005;66(6 Suppl 1):4-34.

[2] Pashos CL, Botteman MF, Laskin BL, Redaelli A. Bladder cancer: epidemiology, diagnosis, and management. Cancer Pract. 2002;10(6):311-22.

[3] Cheng L, Neumann RM, Nehra A, Spotts BE, Weaver AL, Bostwick DG. Cancer heterogeneity and its biologic implications in the grading of urothelial carcinoma. Cancer. 2000;88(7):1663-70.

[4] Zhang Y, Zhu C, Curado MP, Zheng T, Boyle P. Changing patterns of bladder cancer in the USA: evidence of heterogeneous disease. BJU Int. 2012;109(1):52-6.

[5] Chen RC, Shipley WU, Efstathiou JA, Zietman AL. Trimodality bladder preservation therapy for muscle-invasive bladder cancer. J Natl Compr Canc Netw. 2013;11(8):95260 .

[6] Mezei G, Chung F. Return hospital visits and hospital readmissions after ambulatory surgery. Ann Surg. 1999;230(5):721-7.

[7] Patel HD, Ball MW, Cohen JE, Kates M, Pierorazio PM, Allaf ME. Morbidity of urologic surgical procedures: an analysis of rates, risk factors, and outcomes. Urology. 2015;85(3):552-9.

[8] Nieder AM, Meinbach DS, Kim SS, Soloway MS. Transurethral bladder tumor resection: intraoperative and postoperative complications in a residency setting. J Urol. 2005;174(6):2307-9.

[9] Gregg JR, McCormick B, Wang L, Cohen P, Sun D, Penson $\mathrm{DF}$, et al. Short term complications from transurethral resection of bladder tumor. Can J Urol. 2016;23(2):8198-203.

[10] Matulewicz RS, Sharma V, McGuire BB, Oberlin DT, Perry KT, Nadler RB. The effect of surgical duration of transurethral resection of bladder tumors on postoperative complications: An analysis of ACS NSQIP data. Urol Oncol. 2015;33(8):338.e19-24.

[11] Ghali F, Moses RA, Raffin E, Hyams ES. What factors are associated with unplanned return following transurethral resection of bladder tumor? An analysis of a large single institution's experience. Scand J Urol. 2016;50(5):370-3.

[12] Hollenbeck BK, Miller DC, Taub D, Dunn RL, Khuri SF, Henderson WG, et al. Risk factors for adverse outcomes after transurethral resection of bladder tumors. Cancer. 2006;106(7):1527-35. 